\title{
Apolipoprotein C-III and its defined lipoprotein subspecies in relation to incident diabetes: the Multi-Ethnic Study of Atherosclerosis
}

\author{
Sarah A. Aroner ${ }^{1}$ - Jeremy D. Furtado ${ }^{1}$. Frank M. Sacks ${ }^{1,2} \cdot$ Michael Y. Tsai $^{3} \cdot$ Kenneth J. Mukamal $^{4}$. \\ Robyn L. McClelland ${ }^{5}$ - Majken K. Jensen ${ }^{1,2}$
}

Received: 22 June 2018 / Accepted: 7 February 2019 / Published online: 4 April 2019

(C) Springer-Verlag GmbH Germany, part of Springer Nature 2019

\begin{abstract}
Aims/hypothesis Apolipoprotein C-III (apoC-III) is a small proinflammatory protein that may play a key role in diabetes pathophysiology. However, prior observational studies have been limited to predominantly white populations, and the biological links between apoC-III and diabetes, particularly the role of apoC-III on specific lipoprotein particles, are not yet well understood. We therefore investigated associations of total apoC-III and apoC-III-defined lipoprotein subspecies with incident diabetes and glucose metabolism measures in a multi-ethnic cohort.

Methods For the current analyses, baseline (2000-2002) plasma total apoC-III and apolipoprotein A-I concentrations of HDL containing or lacking apoC-III were newly measured via sandwich ELISA in 4579 participants from the Multi-Ethnic Study of Atherosclerosis. Multivariable Cox regression was used to examine associations of apolipoproteins with incident diabetes until early 2012 (567 cases), and linear mixed models were used to estimate associations with longitudinally assessed continuous measures of glucose metabolism. Similar exploratory analyses of plasma apolipoprotein B concentrations of LDL and VLDL containing or lacking apoC-III were performed in a subset of participants (LDL, $n=1545$; VLDL, $n=1526$ ).

Results In the overall population, elevated total apoC-III concentrations were associated with a higher rate of diabetes (top vs bottom quintile, HR 1.88; $95 \%$ CI 1.42, 2.47; $\left.p_{\text {trend }}=0.0002\right)$. ApoC-III-defined HDL subspecies displayed opposing associations with incidence of diabetes $(p$ for heterogeneity $=0.02$ ). While HDL lacking apoC-III was inversely associated with incidence of diabetes (top vs bottom quintile, HR 0.66; 95\% CI 0.46, 0.93; $p_{\text {trend }}=0.002$ ), HDL containing apoC-III was not associated (HR 1.11; $95 \%$ CI $\left.0.78,1.58 ; p_{\text {trend }}=0.61\right)$. Similarly, only HDL lacking apoC-III was beneficially associated with plasma glucose $\left(p_{\text {trend }}=0.003\right), \mathrm{HbA}_{1 \mathrm{c}}\left(p_{\text {trend }}=0.04\right)$ and insulin sensitivity $\left(p_{\text {trend }}<0.0001\right)$, and higher HDL containing apoCIII was associated with lower insulin sensitivity $\left(p_{\text {trend }}=0.04\right)$. Neither of the apoC-III-defined LDL subspecies was associated with incident diabetes, while VLDL was more strongly associated with the incidence of diabetes when it lacked apoC-III. Further adjustment for plasma triacylglycerols as a potential intermediate attenuated the associations of total apoC-III and apoC-IIIdefined lipoprotein subspecies. No statistically significant differences were observed across racial/ethnic groups.

Conclusions/interpretation Our findings in a multi-ethnic population support the involvement of apoC-III in the development of diabetes, potentially through its association with circulating triacylglycerols. The presence of apoC-III on HDL also diminished the protective association of HDL with incident diabetes. Further investigation of apoC-III and apoC-III-defined HDL subspecies may inform the development of novel diabetes treatment and prevention strategies.
\end{abstract}

Electronic supplementary material The online version of this article (https://doi.org/10.1007/s00125-019-4847-8) contains peer-reviewed but unedited supplementary material, which is available to authorised users.

Sarah A. Aroner

saroner@mail.harvard.edu

1 Department of Nutrition, Harvard T.H. Chan School of Public Health, 655 Huntington Avenue, Boston, MA 02115, USA

2 Channing Division of Network Medicine, Department of Medicine, Brigham and Women's Hospital and Harvard Medical School, Boston, MA, USA
3 Department of Laboratory Medicine and Pathology, University of Minnesota, Minneapolis, MN, USA

4 Division of General Medicine and Primary Care, Beth Israel Deaconess Medical Center, Boston, MA, USA

5 Department of Biostatistics, University of Washington, Seattle, WA, USA 


\section{Research in context}

\section{What is already known about this subject?}

- Apolipoprotein C-III (apoC-III) is important in cardiovascular disease, and emerging evidence suggests that it may also be involved in the pathophysiology of diabetes through lipoprotein metabolism, pancreatic beta cell apoptosis and glucose homoeostasis

- Limited observational evidence from predominantly white populations supports a positive association between circulating apoC-III concentrations and risk of diabetes

\section{What is the key question?}

- Is plasma apoC-III itself associated with a higher rate of diabetes, and does the presence of apoC-III on lipoprotein particles influence their associations with incident diabetes in a multi-ethnic population?

\section{What are the new findings?}

- In a cohort including individuals of four racial/ethnic groups, plasma apoC-III concentrations were positively associated with incident diabetes and adversely associated with glucose metabolism measures

- $\quad \mathrm{HDL}$ lacking apoC-III (but not HDL containing apoC-III) was inversely associated with the incidence of diabetes. ApoCIII-defined LDL subspecies were not associated with incident diabetes

- Measures of plasma triacylglycerol levels were strong intermediates for associations of total apoC-III and apoC-IIIdefined lipoprotein subspecies

How might this impact on clinical practice in the foreseeable future?

- ApoC-III may serve as a novel therapeutic target in diabetes treatment, and measurement of apoC-III-defined HDL subspecies may inform the development of HDL-raising anti-diabetogenic agents

Keywords Apolipoprotein C-III · Biomarkers · Diabetes · Glucose metabolism · Lipoproteins · Observational study

\begin{tabular}{ll}
\multicolumn{2}{l}{ Abbreviations } \\
ApoA-I & Apolipoprotein A-I \\
ApoB & Apolipoprotein B \\
ApoC-III & Apolipoprotein C-III \\
CRP & C-reactive protein \\
CVD & Cardiovascular disease \\
IQR & Interquartile range \\
MESA & Multi-Ethnic Study of Atherosclerosis \\
Mffm/I & Insulin sensitivity corrected for fat-free mass \\
$p_{\text {het }}$ & $p$ for heterogeneity
\end{tabular}

\section{Introduction}

Diabetes has risen to epidemic proportions, with $9 \%$ of US adults now living with diabetes [1] and the prevalence estimated to be even higher among some racial/ethnic minorities [2]. As current preventive efforts that focus primarily on the management of glucose homeostasis have failed to stem the growing burden of diabetes, preventive strategies targeting novel biological mechanisms are urgently needed. Increasing support for the involvement of lipid-related pathways in the pathophysiology of diabetes [2,3] suggests the particular promise of approaches aimed at markers of dysregulated lipid metabolism.

Substantial evidence implicates the proinflammatory protein apolipoprotein C-III (apoC-III), sometimes present on lipoproteins (HDL, LDL and VLDL), as a risk factor for cardiovascular disease (CVD) [4]. Growing research suggests that apoC-III may also play a critical role in the aetiology of diabetes through a variety of biological mechanisms. ApoCIII is primarily recognised for its ability to upregulate circulating triacylglycerol levels by delaying the removal [5] and inhibiting the lipolysis of triacylglycerol-rich lipoproteins [6-9], potentially contributing to the development of both CVD and diabetes. In addition, apoC-III may exert specific diabetogenic actions that include the impairment of insulin signalling [10], stimulation of pancreatic beta cell apoptosis $[11,12]$ and promotion of inflammation [13, 14]. Consistent with these biological findings, two prior observational studies in predominantly white populations have reported positive associations between total apoC-III concentrations and risk of diabetes $[15,16]$. However, it remains unclear whether these associations extend to more diverse populations.

In addition to its direct role in cardiometabolic disease pathophysiology, apoC-III may modify the functions of the 
lipoproteins on which it resides. We previously demonstrated in several cohorts that the presence of apoC-III diminishes the established inverse association of HDL with CHD [17] and subclinical atherosclerosis [18] and strengthens the positive association of LDL with risk of CHD [19], suggesting that apoC-III may disrupt the cardioprotective functions of HDL and enhance the atherogenicity of LDL. Our findings for cardiovascular outcomes led us to hypothesise that apoC-III may similarly counter the emerging anti-diabetogenic properties of HDL, which include the regulation of glucose and insulin secretion and the suppression of inflammation in metabolic tissues [2]. Although LDL is not considered to be an important risk factor for diabetes, we hypothesised that the presence of apoC-III on LDL might render it diabetogenic, perhaps by amplifying the potential inhibitory effects of LDL on insulin secretion [20]. We also speculated that the presence of apoCIII on VLDL might further strengthen its adverse association with incident diabetes [21,22].

To investigate associations of total apoC-III and apoC-IIIdefined HDL, LDL and VLDL subspecies with incident diabetes, we performed a prospective analysis in the Multi-Ethnic Study of Atherosclerosis (MESA). We additionally evaluated associations of these apolipoprotein exposures with repeated measures of glucose metabolism to provide further insight into their potential involvement in the development of diabetes. Importantly, the racial/ethnic diversity of MESA allowed us to explore these associations in a representative population.

\section{Methods}

Study population and design MESA is a population-based prospective study among 6814 participants aged 45-84 and free of clinical CVD at baseline in 2000-2002 [23]. Participants of European (38\%), African-American (28\%), Hispanic (22\%) and Chinese-American (12\%) descent were recruited from six US communities (Baltimore, MD; Chicago, IL; Forsyth County, NC; Los Angeles County, CA; northern Manhattan, NY; and St Paul, MN). Follow-up examinations occurred in 2002-2003 (Examination 2), 2004-2005 (Examination 3), 2005-2007 (Examination 4) and 20102012 (Examination 5). Informed consent was obtained from all study participants, and the institutional review board at each study site approved the study protocol.

Information on demographic and lifestyle factors was obtained via questionnaire, and anthropomorphic measurements were obtained by trained personnel. Blood biomarkers were measured from fasting blood samples at the MESA central laboratory at the University of Minnesota.

Apolipoprotein measurements Total apoC-III and HDL measures, assessed via concentrations of apolipoprotein A-I (apoA-I) (the main structural protein of HDL), were newly assayed for the purposes of these analyses in baseline plasma samples from 5796 MESA participants (1000 participants were randomly excluded by the MESA Steering Committee to preserve sample volume, and an additional 18 participants had insufficient sample volume for analyses). As LDL and VLDL containing or lacking apoC-III were examined as a secondary aim, baseline concentrations of these subspecies were assayed in a case-cohort subset of 1834 participants, with LDL and VLDL determined via concentrations of apolipoprotein $\mathrm{B}$ (apoB) following ultracentrifugation to isolate VLDL from whole plasma containing LDL. Apolipoprotein measurements were obtained via sandwich ELISA as detailed in the electronic supplementary material (ESM) Methods.

After exclusions for undetectable or implausibly low apolipoprotein values, no follow-up examinations, diabetes at baseline, missing information on diabetes at baseline or on all follow-up examinations, missing covariate information and extreme total apoA-I values, 4579 participants were available for analyses of total apoC-III and apoC-III-defined HDL subspecies in the full cohort. Similar exclusions in the casecohort with apoB measures resulted in 1545 participants for analyses of LDL subspecies and 1526 participants for analyses of VLDL subspecies. Full details regarding these exclusion criteria are provided in the ESM Methods.

\section{Glucose metabolism measures and incident diabetes}

Measures of glucose metabolism were obtained from fasting blood samples. Plasma glucose was measured at baseline and all four follow-up examinations, $\mathrm{HbA}_{1 \mathrm{c}}$ was measured at Examinations 2 and 5, and serum insulin was measured at baseline and Examination 5. Further details are provided in the ESM Methods. Insulin sensitivity corrected for fat-free mass $(\mathrm{Mffm} / \mathrm{I})$ was estimated using an index (exp[2.63$0.28 \log _{e}$ (insulin) $-0.31 \log _{e}$ (triacylglycerols)]) described by McAuley et al [24]. Diabetes was ascertained at all examinations according to the ADA criteria of fasting plasma glucose $\geq 7.0 \mathrm{mmol} / 1$ or use of insulin or oral hypoglycaemic medications [25], with medication use assessed via self-report and medication inventory.

Statistical analyses Baseline participant characteristics were evaluated across quintiles of apoC-III using means (SD) or medians (interquartile range [IQR]) for continuous variables and percentages for categorical variables. Cox proportional hazards models were used to estimate associations between apolipoprotein exposures (total apoC-III, total HDL, total LDL, total VLDL and lipoproteins containing or lacking apoC-III) and incident diabetes. Models used to estimate associations for total VLDL, total LDL and apoC-III-defined VLDL and LDL subtypes included Kalbfleisch and Lawless weights and applied a robust variance estimator to accommodate the case-cohort design used for these analyses [26]. Cases both within and outside of the subcohort were assigned a 
weight of 1 , and non-cases (all within the subcohort) were weighted inversely to their probability of selection into the subcohort.

Apolipoprotein exposures were natural log-transformed to improve normality. The proportional hazards assumption was confirmed through analysis of Schoenfeld residuals. Persontime was calculated as years from the midpoint of the baseline examination to the midpoint of the examination at which diabetes was first identified or censoring occurred due to loss to follow-up, death or the end of follow-up at Examination 5 (2010-2012). The exact method [27] was used to account for the tied event times. Associations were examined across quintiles and per SD of apolipoprotein exposures.

Linear mixed regression models with random intercepts were used to calculate adjusted means for longitudinally assessed continuous glucose metabolism measures according to quintiles and per SD of total apoC-III, total HDL and apoCIII-defined HDL subspecies. Participants with measurements from at least one examination were included in analyses of each outcome measure ( $N=4579$ for fasting plasma glucose, $N=4503$ for $\mathrm{HbA}_{1 \mathrm{c}}$, and $N=4575$ for insulin sensitivity). Wald trend tests for associations with incident diabetes and glucose metabolism measures were performed across $\left(\log _{e^{-}}\right.$ transformed) quintile median apolipoprotein values. Incident treated diabetes cases were censored at the examination at which diabetes medication use was first reported to minimise the influence of treatment on glucose metabolism measures (untreated diabetes cases remained in the analysis). Similar analyses of LDL and VLDL measures in relation to fasting plasma glucose were conducted in the random subcohort.

All models were stratified by age and sex and adjusted for race/ethnicity (white, African-American, Hispanic, ChineseAmerican). Multivariable models were additionally adjusted for smoking, income, alcohol intake, BMI, systolic BP and anti-hypertensive medication use. Associations of these covariates, as well as triacylglycerol levels, with incident diabetes and plasma glucose in the multivariable models are presented in ESM Table 1. Results were essentially identical with adjustment for waist circumference instead of or in addition to BMI, and estimates were also minimally influenced with adjustment for physical activity (total moderate and vigorous activity), field centre and dietary variables (polyunsaturated fat, saturated fat, trans fat, sugar-sweetened beverage intake, total vegetable intake, total fibre and total energy intake) (ESM Table 2). We assessed the heterogeneity between associations for the two lipoprotein subspecies with diabetes incidence and glucose metabolism measures in models with both subspecies (e.g. HDL containing or lacking apoC-III) included separately as continuous quintile medians. We then tested the hypothesis that the regression coefficients for the two subfractions were equal (using a $1 d f$ Wald test).

As we hypothesised that apoC-III may contribute to the development of diabetes by enhancing triacylglycerol levels
$[6,28]$ or promoting inflammation [13], we further adjusted for (log-transformed) triacylglycerol levels and inflammatory markers (C-reactive protein [CRP] and IL-6) to determine whether these pathways might explain associations of total apoC-III and apoC-III-defined lipoprotein subspecies with incidence of diabetes. We adjusted for total HDL, total LDL and total VLDL in additional models to assess whether apoC-III might contribute to incident diabetes beyond established lipid measures. We also examined associations for LDL measures after excluding lipid-lowering medication users at baseline and censoring for lipid-lowering medication use over followup. As results were essentially unchanged in these sensitivity analyses, we did not account for lipid-lowering medication use in our final models.

We additionally examined associations of total apoC-III and HDL subspecies with incident diabetes in strata of sex, race (white vs non-white), BMI $\left(<25 \mathrm{~kg} / \mathrm{m}^{2}, \geq 25 \mathrm{~kg} / \mathrm{m}^{2}\right)$, smoking (non-smoking, smoking) and presence of the metabolic syndrome and tested for interaction by these variables using likelihood ratio tests. The metabolic syndrome was defined according to the National Cholesterol Education Program's Adult Treatment Panel III guidelines as meeting three out of five of the following criteria: abdominal obesity, hypertriacylglycerolaemia, low HDL-cholesterol, high BP and high fasting glucose [29].

\section{Results}

Over a median follow-up period of 9.6 years, 567 cases occurred among the 4579 study participants. The mean age of study participants was 62 years at baseline. Forty-one per cent of participants were white, $12 \%$ were Chinese-American, $27 \%$ were African-American and $21 \%$ were Hispanic. The median percentages of HDL, LDL and VLDL containing apoC-III were $6.3 \%, 2.9 \%$ and $10.1 \%$, respectively. Total apoC-III levels were higher among postmenopausal women, lipidlowering medication users and current alcohol consumers (Table 1). HDL and LDL containing apoC-III were inversely correlated with BMI and waist circumference, while VLDL containing apoC-III was positively correlated. Total apoC-III and both LDL and VLDL containing apoC-III displayed a strong positive correlation with plasma triacylglycerols, while HDL containing apoC-III was uncorrelated (ESM Table 3). Levels of total apoC-III and apoC-III-defined lipoprotein subspecies were similar across racial/ethnic groups (ESM Table 4).

Associations of total apoC-III with incident diabetes and glucose metabolism measures No statistically significant interactions were observed for apoC-III with sex or race/ethnicity. Therefore, all analyses were performed in the overall MESA population with sex and race/ethnicity accounted for in 
Table 1 Baseline characteristics by quintiles of apoC-III in MESA $(N=4579)$

\begin{tabular}{|c|c|c|c|c|c|}
\hline Characteristic & $\begin{array}{l}\text { Q1 } \\
(n=916)\end{array}$ & $\begin{array}{l}\text { Q2 } \\
(n=916)\end{array}$ & $\begin{array}{l}\text { Q3 } \\
(n=916)\end{array}$ & $\begin{array}{l}\text { Q4 } \\
(n=916)\end{array}$ & $\begin{array}{l}\text { Q5 } \\
(n=915)\end{array}$ \\
\hline Quintile median (range), g/l & $\begin{array}{l}0.053 \\
(\leq 0.064)\end{array}$ & $\begin{array}{l}0.073 \\
(0.064-0.079)\end{array}$ & $\begin{array}{l}0.086 \\
(0.079-0.093)\end{array}$ & $\begin{array}{l}0.103 \\
(0.093-0.115)\end{array}$ & $\begin{array}{l}0.136 \\
(0.115-0.516)\end{array}$ \\
\hline Age, mean years (SD) & $61.5(10.8)$ & $62.5(10.6)$ & $62.0(10.1)$ & $62.3(10.0)$ & $62.0(9.8)$ \\
\hline Female, N (\%) & $362(39.5)$ & $425(46.4)$ & $495(54.0)$ & $549(59.9)$ & $562(61.4)$ \\
\hline Postmenopausal, $N(\%)^{\mathrm{a}}$ & $250(69.3)$ & $343(80.9)$ & $409(82.6)$ & $456(83.2)$ & $498(88.6)$ \\
\hline \multicolumn{6}{|l|}{ Race/ethnicity, $N(\%)$} \\
\hline White & $274(29.9)$ & $331(36.1)$ & $361(39.4)$ & $425(46.4)$ & $476(52.0)$ \\
\hline Chinese-American & $78(8.5)$ & $115(12.6)$ & $124(13.5)$ & $123(13.4)$ & $115(12.6)$ \\
\hline African-American & $389(42.5)$ & $286(31.2)$ & $256(28.0)$ & $174(19.0)$ & $110(12.0)$ \\
\hline Hispanic & $175(19.1)$ & $184(20.1)$ & $175(19.1)$ & $194(21.2)$ & $214(23.4)$ \\
\hline Education less than high school, $N(\%)$ & $285(31.1)$ & $296(32.3)$ & $293(32.0)$ & $322(35.2)$ & $337(36.8)$ \\
\hline Income $<\$ 25,000 /$ year, $N(\%)^{\mathrm{b}}$ & $251(28.6)$ & $250(28.4)$ & $253(28.4)$ & $262(29.5)$ & $288(32.3)$ \\
\hline Hypertension, $N(\%)$ & $370(40.4)$ & $345(37.7)$ & $387(42.3)$ & $393(42.9)$ & $415(45.4)$ \\
\hline Hypercholesterolaemia, $N(\%)$ & $22(2.4)$ & $38(4.2)$ & $75(8.2)$ & $107(11.7)$ & $191(20.9)$ \\
\hline Use of anti-hypertensive medication, $N(\%)$ & $300(32.8)$ & $264(28.8)$ & $314(34.3)$ & $311(34.0)$ & $334(36.5)$ \\
\hline Use of lipid-lowering medication, $N(\%)$ & $102(11.1)$ & $124(13.5)$ & $132(14.4)$ & $136(14.9)$ & $205(22.4)$ \\
\hline Systolic BP, mean mmHg (SD) & $124.8(21.2)$ & $125.0(20.8)$ & $124.8(20.9)$ & $126.7(20.7)$ & $126.9(21.3)$ \\
\hline BMI, mean kg/m² (SD) & $28.1(5.5)$ & $27.7(5.2)$ & $27.7(5.4)$ & $27.9(5.1)$ & $28.3(5.1)$ \\
\hline Waist circumference, mean cm (SD) & $97.1(14.7)$ & $96.5(13.7)$ & $96.3(14.0)$ & $97.5(13.8)$ & $98.3(13.6)$ \\
\hline $\begin{array}{l}\text { Moderate and vigorous physical activity, } \\
\text { median MET } \mathrm{h} / \text { week (IQR) }{ }^{\mathrm{c}}\end{array}$ & $\begin{array}{l}54 \\
(29-95)\end{array}$ & $\begin{array}{l}65 \\
(34-110)\end{array}$ & $\begin{array}{l}63 \\
(31-106)\end{array}$ & $\begin{array}{l}61 \\
(30-98)\end{array}$ & $\begin{array}{l}60 \\
(31-104)\end{array}$ \\
\hline Current smoker, $N(\%)$ & $136(14.9)$ & $112(12.2)$ & $107(11.7)$ & $104(11.4)$ & $112(12.2)$ \\
\hline Current alcohol intake, $N(\%)$ & $485(53.0)$ & $512(55.9)$ & $530(57.9)$ & $554(60.5)$ & $581(63.5)$ \\
\hline $\begin{array}{l}\text { Triacylglycerols, } \\
\text { median } \mathrm{mmol} / \mathrm{l}(\mathrm{IQR})\end{array}$ & $\begin{array}{l}0.8 \\
(0.6-1.0)\end{array}$ & $\begin{array}{l}1.1 \\
(0.8-1.3)\end{array}$ & $\begin{array}{l}1.2 \\
(0.9-1.6)\end{array}$ & $\begin{array}{l}1.5 \\
(1.1-2.0)\end{array}$ & $\begin{array}{l}2.1 \\
(1.5-2.8)\end{array}$ \\
\hline $\begin{array}{l}\text { HDL-C, } \\
\text { median mmol/l (IQR) }\end{array}$ & $\begin{array}{l}1.2 \\
(1.0-1.4)\end{array}$ & $\begin{array}{l}1.3 \\
(1.1-1.5)\end{array}$ & $\begin{array}{l}1.3 \\
(1.1-1.6)\end{array}$ & $\begin{array}{l}1.3 \\
(1.1-1.6)\end{array}$ & $\begin{array}{l}1.2 \\
(1.0-1.5)\end{array}$ \\
\hline $\begin{array}{l}\text { Plasma glucose, } \\
\text { median mmol/l (IQR) }\end{array}$ & $\begin{array}{l}4.9 \\
(4.5-5.3)\end{array}$ & $\begin{array}{l}4.9 \\
(4.6-5.3)\end{array}$ & $\begin{array}{l}4.9 \\
(4.6-5.2)\end{array}$ & $\begin{array}{l}4.9 \\
(4.6-5.3)\end{array}$ & $\begin{array}{l}5.0 \\
(4.6-5.4)\end{array}$ \\
\hline \multicolumn{6}{|l|}{$\mathrm{HbA}_{1 \mathrm{c}}{ }^{\mathrm{d}}$} \\
\hline Median mmol/mol (IQR) & $\begin{array}{l}36 \\
(33-39)\end{array}$ & $\begin{array}{l}37 \\
(33-39)\end{array}$ & $\begin{array}{l}36 \\
(32-39)\end{array}$ & $\begin{array}{l}36 \\
(33-39)\end{array}$ & $\begin{array}{l}36 \\
(33-39)\end{array}$ \\
\hline Median \% (IQR) & $\begin{array}{l}5.4 \\
(5.2-5.7)\end{array}$ & $\begin{array}{l}5.5 \\
(5.2-5.7)\end{array}$ & $\begin{array}{l}5.4 \\
(5.1-5.7)\end{array}$ & $\begin{array}{l}5.4 \\
(5.2-5.7)\end{array}$ & $\begin{array}{l}5.4 \\
(5.2-5.7)\end{array}$ \\
\hline $\begin{array}{l}\text { Insulin sensitivity, } \\
\text { median Mffm/I (IQR) }\end{array}$ & $\begin{array}{l}2.1 \\
(1.8-2.4)\end{array}$ & $\begin{array}{l}1.9 \\
(1.7-2.2)\end{array}$ & $\begin{array}{l}1.8 \\
(1.6-2.1)\end{array}$ & $\begin{array}{l}1.7 \\
(1.4-2.0)\end{array}$ & $\begin{array}{l}1.5 \\
(1.3-1.8)\end{array}$ \\
\hline $\begin{array}{l}\text { Total apoA-I in HDL, } \\
\text { median } \mathrm{g} / \mathrm{l}(\mathrm{IQR})\end{array}$ & $\begin{array}{l}1.1 \\
(1.0-1.3)\end{array}$ & $\begin{array}{l}1.2 \\
(1.1-1.4)\end{array}$ & $\begin{array}{l}1.3 \\
(1.1-1.5)\end{array}$ & $\begin{array}{l}1.3 \\
(1.2-1.6)\end{array}$ & $\begin{array}{l}1.4 \\
(1.2-1.7)\end{array}$ \\
\hline $\begin{array}{l}\text { ApoA-I in HDL containing apoC-III, } \\
\text { median } g / 1 \text { (IQR) }\end{array}$ & $\begin{array}{l}0.06 \\
(0.05-0.08)\end{array}$ & $\begin{array}{l}0.08 \\
(0.06-0.09)\end{array}$ & $\begin{array}{l}0.08 \\
(0.07-0.10)\end{array}$ & $\begin{array}{l}0.09 \\
(0.07-0.11)\end{array}$ & $\begin{array}{l}0.10 \\
(0.08-0.12)\end{array}$ \\
\hline $\begin{array}{l}\text { ApoA-I in HDL lacking apoC-III, } \\
\text { median g/l (IQR) }\end{array}$ & $\begin{array}{l}1.1 \\
(0.9-1.2)\end{array}$ & $\begin{array}{l}1.2 \\
(1.0-1.3)\end{array}$ & $\begin{array}{l}1.2 \\
(1.0-1.4)\end{array}$ & $\begin{array}{l}1.3 \\
(1.1-1.5)\end{array}$ & $\begin{array}{l}1.3 \\
(1.1-1.6)\end{array}$ \\
\hline $\begin{array}{l}\text { Percentage of apoA-I containing apoC-III, } \\
\text { median \% (IQR) }\end{array}$ & $\begin{array}{l}5.70 \\
(4.82-6.58)\end{array}$ & $\begin{array}{l}6.15 \\
(5.35-6.95)\end{array}$ & $\begin{array}{l}6.32 \\
(5.48-7.18)\end{array}$ & $\begin{array}{l}6.60 \\
(5.76-7.46)\end{array}$ & $\begin{array}{l}6.83 \\
(5.91-7.97)\end{array}$ \\
\hline $\begin{array}{l}\text { Total apoB in LDL, } \\
\text { median } \mathrm{g} / 1(\mathrm{IQR})^{\mathrm{e}}\end{array}$ & $\begin{array}{l}0.650 \\
(0.546-0.764)\end{array}$ & $\begin{array}{l}0.720 \\
(0.610-0.811)\end{array}$ & $\begin{array}{l}0.726 \\
(0.609-0.852)\end{array}$ & $\begin{array}{l}0.753 \\
(0.648-0.859)\end{array}$ & $\begin{array}{l}0.759 \\
(0.633-0.910)\end{array}$ \\
\hline $\begin{array}{l}\text { ApoB in LDL containing apoC-III, } \\
\text { median } g / 1(\mathrm{IQR})^{\mathrm{e}}\end{array}$ & $\begin{array}{l}0.013 \\
(0.010-0.018)\end{array}$ & $\begin{array}{l}0.018 \\
(0.015-0.022)\end{array}$ & $\begin{array}{l}0.020 \\
(0.017-0.025)\end{array}$ & $\begin{array}{l}0.024 \\
(0.019-0.028)\end{array}$ & $\begin{array}{l}0.031 \\
(0.024-0.039)\end{array}$ \\
\hline $\begin{array}{l}\text { ApoB in LDL lacking apoC-III, } \\
\text { median } \mathrm{g} / \mathrm{l}(\mathrm{IQR})^{\mathrm{e}}\end{array}$ & $\begin{array}{l}0.637 \\
(0.532-0.746)\end{array}$ & $\begin{array}{l}0.697 \\
(0.594-0.795)\end{array}$ & $\begin{array}{l}0.705 \\
(0.591-0.829)\end{array}$ & $\begin{array}{l}0.728 \\
(0.623-0.832)\end{array}$ & $\begin{array}{l}0.731 \\
(0.608-0.882)\end{array}$ \\
\hline
\end{tabular}


Table 1 (continued)

\begin{tabular}{|c|c|c|c|c|c|}
\hline Characteristic & $\begin{array}{l}\text { Q1 } \\
(n=916)\end{array}$ & $\begin{array}{l}\text { Q2 } \\
(n=916)\end{array}$ & $\begin{array}{l}\text { Q3 } \\
(n=916)\end{array}$ & $\begin{array}{l}\text { Q4 } \\
(n=916)\end{array}$ & $\begin{array}{l}\text { Q5 } \\
(n=915)\end{array}$ \\
\hline $\begin{array}{l}\text { Percentage of apoB in LDL containing } \\
\text { apoC-III, median } \%(\mathrm{IQR})^{\mathrm{e}}\end{array}$ & $\begin{array}{l}2.05 \\
(1.56-2.67)\end{array}$ & $\begin{array}{l}2.51 \\
(2.05-3.09)\end{array}$ & $\begin{array}{l}2.81 \\
(2.32-3.40)\end{array}$ & $\begin{array}{l}3.28 \\
(2.61-3.91)\end{array}$ & $\begin{array}{l}4.00 \\
(3.25-5.07)\end{array}$ \\
\hline $\begin{array}{l}\text { Total apoB in VLDL, } \\
\text { median } \mathrm{g} / \mathrm{l}(\mathrm{IQR})^{\mathrm{f}}\end{array}$ & $\begin{array}{l}0.005 \\
(0.003-0.009)\end{array}$ & $\begin{array}{l}0.009 \\
(0.004-0.014)\end{array}$ & $\begin{array}{l}0.011 \\
(0.005-0.017)\end{array}$ & $\begin{array}{l}0.015 \\
(0.008-0.022)\end{array}$ & $\begin{array}{l}0.019 \\
(0.013-0.031)\end{array}$ \\
\hline $\begin{array}{l}\text { ApoB in VLDL containing apoC-III, } \\
\text { median } g / 1(\mathrm{IQR})^{\mathrm{f}}\end{array}$ & $\begin{array}{l}0.0006 \\
(0.0004-0.0009)\end{array}$ & $\begin{array}{l}0.0008 \\
(0.0005-0.0013)\end{array}$ & $\begin{array}{l}0.0010 \\
(0.0006-0.0015)\end{array}$ & $\begin{array}{l}0.0013 \\
(0.0008-0.0019)\end{array}$ & $\begin{array}{l}0.0017 \\
(0.0012-0.0026)\end{array}$ \\
\hline 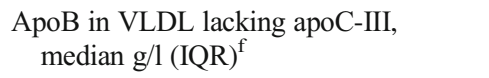 & $\begin{array}{l}0.005 \\
(0.002-0.008)\end{array}$ & $\begin{array}{l}0.008 \\
(0.004-0.013)\end{array}$ & $\begin{array}{l}0.010 \\
(0.005-0.015)\end{array}$ & $\begin{array}{l}0.013 \\
(0.007-0.019)\end{array}$ & $\begin{array}{l}0.017 \\
(0.012-0.027)\end{array}$ \\
\hline $\begin{array}{l}\text { Percentage of apoB in VLDL containing } \\
\text { apoC-III, median \% (IQR) }\end{array}$ & $\begin{array}{l}11.17 \\
(7.14-16.21)\end{array}$ & $\begin{array}{l}10.06 \\
(7.19-14.19)\end{array}$ & $\begin{array}{l}10.35 \\
(7.50-14.43)\end{array}$ & $\begin{array}{l}9.76 \\
(7.38-13.53)\end{array}$ & $\begin{array}{l}9.80 \\
(6.85-13.91)\end{array}$ \\
\hline
\end{tabular}

${ }^{\text {a }}$ Per cent postmenopausal women calculated from the total number of women with menopausal status data ( $n=1$ missing for Q1, Q2 and Q4)

${ }^{\mathrm{b}}$ Per cent with income $<\$ 25,000 /$ year calculated from the total number of participants with income data $(n=38$ missing for Q1, $n=36$ for Q2, $n=26$ for Q3, $n=28$ for Q4 and $n=23$ for Q5)

${ }^{\mathrm{c}}$ Participants reporting more than $18 \mathrm{~h} /$ day of total physical activity excluded ( $n=3947$ after exclusions)

${ }^{\mathrm{d}} \mathrm{HbA}_{1 \mathrm{c}}$ from Examination 2 due to lack of baseline measurements ( $n=4405$ with Examination $2 \mathrm{HbA}_{1 \mathrm{c}}$ measurements)

e $n=1545$

${ }^{\mathrm{f}} n=1526$

HDL-C, HDL-cholesterol; MET, metabolic equivalents

adjusted models (additional details are presented in the section on effect modification). In multivariable models, participants in the top quintile of total apoC-III had a nearly twofold higher incidence of diabetes compared with those in the bottom quintile (top vs bottom quintile, HR 1.88; 95\% CI 1.42, 2.47; $p_{\text {trend }}=$ 0.0002) (Fig. 1). Total apoC-III was also strongly associated with

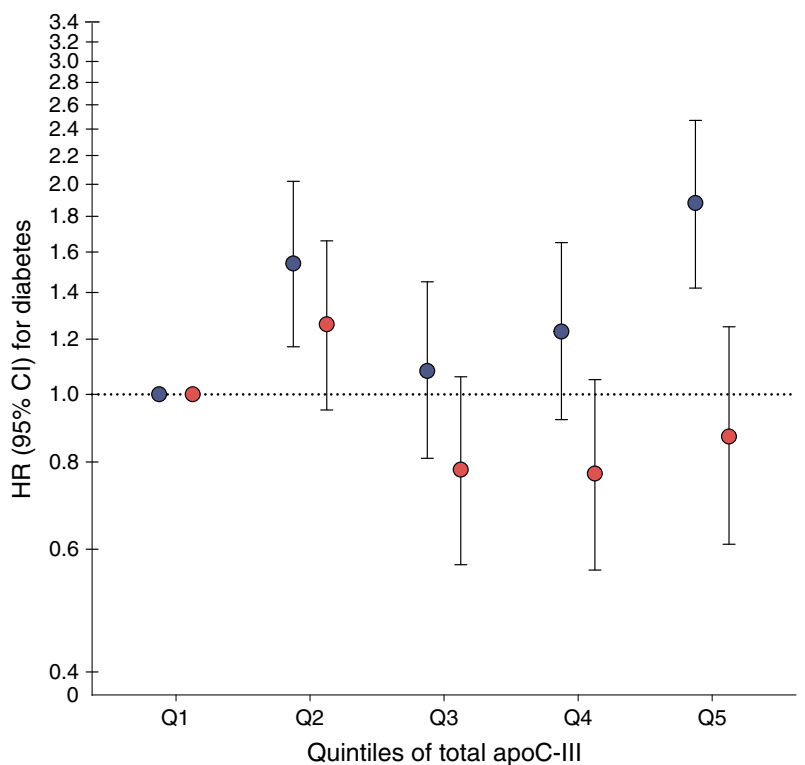

Fig. 1 Incidence of diabetes according to quintiles of total apoC-III in MESA. HRs and $95 \%$ CIs (plotted on a $\log _{10}$ scale) estimated in Cox proportional hazards models stratified by age and sex and adjusted for race, smoking, income, alcohol intake, BMI, systolic BP and anti-hypertensive medication use. Total apoC-III (blue circles), $p_{\text {trend }}=0.0002, \mathrm{HR}$ per $\mathrm{SD}=1.21(95 \% \mathrm{CI} 1.11,1.32)$; total apoC-III + triacylglycerols (red circles), $p_{\text {trend }}=0.12, \mathrm{HR}$ per $\mathrm{SD}=0.92(95 \% \mathrm{CI} 0.81,1.03)$ measures of glucose metabolism, positively with fasting plasma glucose and $\mathrm{HbA}_{1 \mathrm{c}}$, and inversely with insulin sensitivity ( $p_{\text {trend }}$ for all measures <0.001) (Fig. 2).

Associations for apoC-III-defined HDL subspecies and total HDL ApoC-III-defined HDL subspecies were differentially associated with incident diabetes (multivariable $p$ for heterogeneity $\left[p_{\text {het }}\right]$, HDL containing and lacking apoC-III $=0.02$ ) (Table 2). HDL lacking apoC-III was inversely associated with the incidence of diabetes (top vs bottom quintile, HR $0.66 ; 95 \%$ CI $0.46,0.93 ; p_{\text {trend }}=0.002$ ), more strongly than total HDL (top vs bottom quintile, HR 0.72; 95\% CI 0.53 , $\left.0.98 ; p_{\text {trend }}=0.003\right)$. In contrast, HDL containing apoC-III was not associated with incident diabetes (top vs bottom quintile, HR $1.11 ; 95 \%$ CI $\left.0.78,1.58 ; p_{\text {trend }}=0.61\right)$.

Higher levels of HDL lacking apoC-III were associated with lower plasma glucose $\left(p_{\text {trend }}=0.003\right)$ and $\mathrm{HbA}_{1 \mathrm{c}}\left(p_{\text {trend }}=0.04\right)$ and higher insulin sensitivity $\left(p_{\text {trend }}<0.0001\right)$, similar to the associations for total HDL (Fig. 2). HDL containing apoC-III was not associated with glucose $\left(p_{\text {trend }}=0.97\right)$ or $\mathrm{HbA}_{1 \mathrm{c}}$ $\left(p_{\text {trend }}=0.95\right)$ and was inversely associated with insulin sensitivity $\left(p_{\text {trend }}=0.04\right)$. The difference in associations for the two apoC-III-defined HDL subspecies was borderline significant for glucose $\left(p_{\text {het }}=0.05\right)$ and highly significant for insulin sensitivity $\left(p_{\text {het }}<0.0001\right)$, but was not significant for $\mathrm{HbA}_{1 \mathrm{c}}\left(p_{\text {het }}=0.17\right)$.

Associations for LDL and VLDL measures In the case-cohort subset with LDL and VLDL measures, no association with diabetes was found for total LDL or for either of the LDL 


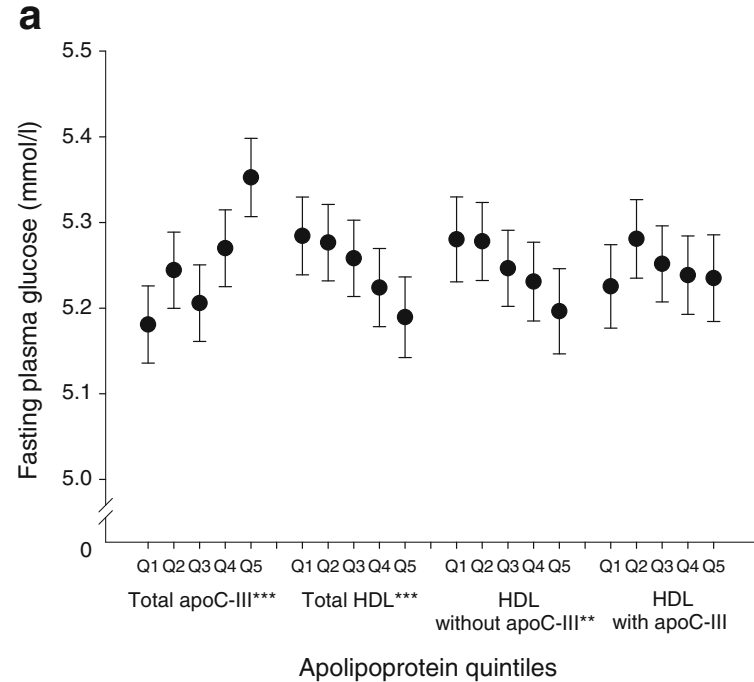

C

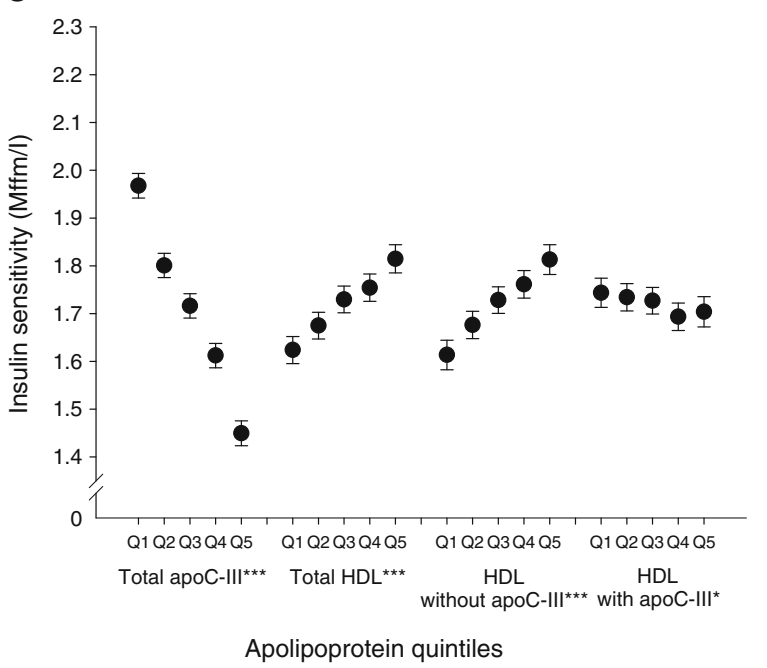

b

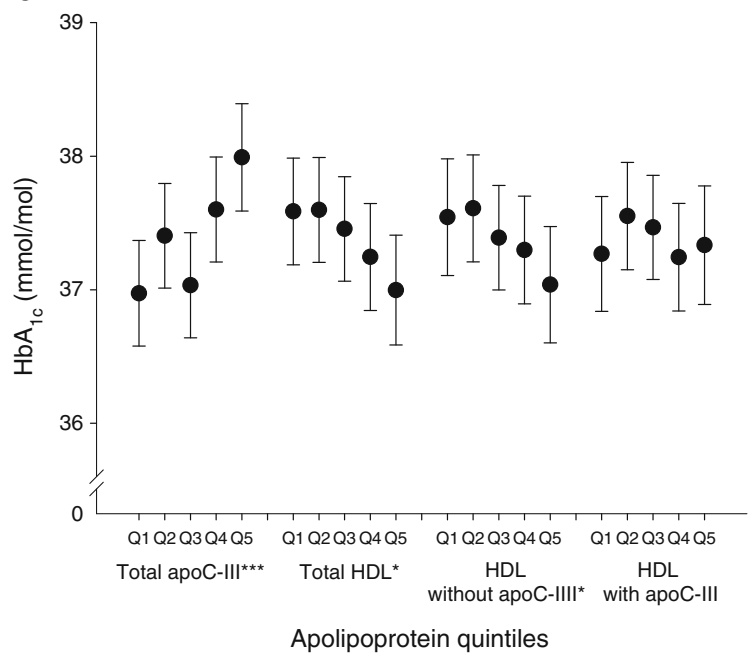

Fig. 2 Adjusted mean values with $95 \%$ CI for (a) fasting plasma glucose (mmol/l), (b) $\mathrm{HbA}_{1 \mathrm{c}}(\mathrm{mmol} / \mathrm{mol})$ and (c) insulin sensitivity $(\mathrm{Mffm} / \mathrm{I})$ in quintiles of total apoC-III, total HDL, HDL without apoC-III and HDL with apoC-III in MESA (HDL assessed via concentrations of apoA-I). Estimates obtained from mixed models adjusted for age, sex, race, smoking, income, alcohol intake, BMI, systolic BP and anti-hypertensive

subspecies in multivariable models (Table 3). Associations with fasting plasma glucose were similarly null for total LDL and apoC-III-defined LDL subspecies in the random subcohort.

Total VLDL displayed a non-significant positive association with incident diabetes (HR per SD 1.10; 95\% CI 0.97, 1.24) (Table 3) and fasting plasma glucose (ESM Fig. 1). Although associations with incident diabetes were not significantly different for the two VLDL subspecies, the positive association for total VLDL appeared to be primarily attributable to VLDL lacking apoC-III (Table 3). A similar pattern was observed for associations of VLDL measures with fasting glucose, with differences in the associations between the two subspecies approaching significance ( $p_{\text {het }}$, VLDL containing or lacking apoC-III =0.05) (ESM Fig. 1a). medication use. The $p_{\text {trend }}$ uses medians of $\log _{e}$-transformed apolipoprotein variables; $p_{\text {het }}$ for HDL containing and lacking apoC-III calculated based on median trends. $p_{\text {het }}$, fasting plasma glucose $=0.05 ; p_{\text {het }}$, $\mathrm{HbA}_{1 \mathrm{c}}=0.17 ; p_{\text {het }}$, insulin sensitivity $<0.0001 .{ }^{*} p_{\text {trend }}<0.05 ; * * p_{\text {trend }}$ $<0.01 ; * * * p_{\text {trend }}<0.001$

Adjustment for triacylglycerols, total lipoproteins and inflammatory markers The strong positive association for total apoC-III with incident diabetes was eliminated with adjustment for plasma triacylglycerols (top vs bottom quintile, HR $0.87 ; 95 \%$ CI $0.61,1.25 ; p_{\text {trend }}=0.12$ ) (Fig. 1). Similarly, the inverse association with diabetes for HDL lacking apoC-III became non-significant with adjustment for triacylglycerols (top vs bottom quintile, HR 0.84; 95\% CI 0.59, 1.20), and differences between the two HDL subspecies were no longer present $\left(p_{\text {het }}=0.61\right)$. Similar attenuation was observed with triacylglycerol adjustment in analyses of continuous glucose metabolism measures. Associations for total LDL and LDL subspecies remained null, and the positive associations for VLDL lacking apoC-III with fasting plasma glucose (ESM 
Table 2 HRs and 95\% CIs for incident diabetes according to plasma concentrations of total HDL and apoC-III-defined HDL subspecies in MESA

\begin{tabular}{|c|c|c|c|c|c|c|c|}
\hline Apolipoprotein exposure & Q1 & Q2 & Q3 & Q4 & Q5 & $p_{\text {trend }}$ & Per $\mathrm{SD}^{\mathrm{b}}$ \\
\hline \multicolumn{8}{|l|}{ Total apoA-I in HDL } \\
\hline $\begin{array}{l}\text { Quintile median, } \\
\text { g/l (range) }\end{array}$ & $\begin{array}{l}0.94 \\
(0.62-1.05)\end{array}$ & $\begin{array}{l}1.14 \\
(1.05-1.21)\end{array}$ & $\begin{array}{l}1.27 \\
(1.21-1.35)\end{array}$ & $\begin{array}{l}1.44 \\
(1.35-1.57)\end{array}$ & $\begin{array}{l}1.76 \\
(1.57-2.55)\end{array}$ & & \\
\hline Crude model & 1.0 (ref) & $1.16(0.92,1.48)$ & $0.92(0.71,1.19)$ & $0.66(0.50,0.87)$ & $0.57(0.43,0.77)$ & $<0.0001$ & $0.81(0.74,0.88)$ \\
\hline Multivariable model & 1.0 (ref) & $1.16(0.91,1.48)$ & $1.00(0.77,1.30)$ & $0.74(0.56,0.99)$ & $0.72(0.53,0.98)$ & 0.003 & $0.87(0.79,0.95)$ \\
\hline \multicolumn{8}{|c|}{ ApoA-I in HDL lacking apoC-III } \\
\hline $\begin{array}{l}\text { Quintile median, } \\
\text { g/l (range) }\end{array}$ & $\begin{array}{l}0.88 \\
(0.56-0.98)\end{array}$ & $\begin{array}{l}1.06 \\
(0.98-1.13)\end{array}$ & $\begin{array}{l}1.19 \\
(1.13-1.26)\end{array}$ & $\begin{array}{l}1.34 \\
(1.26-1.46)\end{array}$ & $\begin{array}{l}1.65 \\
(1.46-2.46)\end{array}$ & & \\
\hline Crude model & 1.0 (ref) & $1.10(0.86,1.41)$ & $0.86(0.65,1.13)$ & $0.63(0.46,0.86)$ & $0.58(0.41,0.83)$ & 0.0001 & $0.85(0.76,0.95)$ \\
\hline Multivariable model & 1.0 (ref) & $1.10(0.86,1.41)$ & $0.90(0.68,1.18)$ & $0.68(0.50,0.93)$ & $0.66(0.46,0.93)$ & 0.002 & $0.88(0.78,0.98)$ \\
\hline \multicolumn{8}{|c|}{ ApoA-I in HDL containing apoC-III } \\
\hline $\begin{array}{l}\text { Quintile median, } \\
\text { g/l (range) }\end{array}$ & $\begin{array}{l}0.051 \\
(0.011-0.060)\end{array}$ & $\begin{array}{l}0.067 \\
(0.060-0.074)\end{array}$ & $\begin{array}{l}0.080 \\
(0.074-0.087)\end{array}$ & $\begin{array}{l}0.095 \\
(0.087-0.107)\end{array}$ & $\begin{array}{l}0.124 \\
(0.107-0.385)\end{array}$ & & \\
\hline Crude model & 1.0 (ref) & $1.08(0.84,1.38)$ & $1.05(0.80,1.37)$ & $1.01(0.75,1.36)$ & $0.95(0.67,1.34)$ & 0.64 & $0.93(0.83,1.04)$ \\
\hline Multivariable model & 1.0 (ref) & $1.07(0.83,1.38)$ & $1.11(0.85,1.46)$ & $1.09(0.81,1.48)$ & $1.11(0.78,1.58)$ & 0.61 & $0.98(0.87,1.10)$ \\
\hline
\end{tabular}

HRs obtained from Cox proportional hazards regression models. Apolipoprotein variables were natural log-transformed in analyses. Quintile median levels displayed above are untransformed. The $p_{\text {trend }}$ was estimated using the medians of (log-transformed) apolipoprotein variables

Crude models stratified by age (years) and sex and adjusted for race (white, African-American, Hispanic, Chinese-American). Multivariable models additionally adjusted for smoking (never, former or current smoker), income $(<\$ 25,000, \$ 25,000-\$ 49,999, \$ 50,000-\$ 74,999$ or $\geq \$ 75,000$ per year), alcohol intake (never, former or current drinker, and current number of drinks per week), BMI (weight $[\mathrm{kg}] / \mathrm{height}[\mathrm{m}]^{2}$ ), systolic BP (mmHg) and antihypertensive medication use

Crude model: $p_{\text {het }}$ (median trend), apoA-I in HDL containing and lacking apoC-III $=0.02$

Multivariable model: $p_{\text {het }}$ (median trend), apoA-I in HDL containing and lacking apoC-III $=0.02$

${ }^{a} \mathrm{HDL}$ was assessed via concentrations of apoA-I, the main structural protein of HDL

${ }^{\mathrm{b}} \mathrm{SD}$ for total apoA-I in HDL, $0.33 \mathrm{~g} / \mathrm{l}$; SD for apoA-I in HDL lacking apoC-III, $0.31 \mathrm{~g} / 1$; SD for apoA-I in HDL containing apoC-III, 0.033 g/1 ref, reference

Fig. 1) and incident diabetes were diminished after adjustment for triacylglycerols. Associations for total apoC-III and incident diabetes became slightly stronger with adjustment for total HDL and were attenuated with adjustment for total VLDL, while adjustment for total LDL had little impact on estimates. Associations for all apolipoproteins were materially unchanged with adjustment for inflammatory markers (CRP and IL-6).

Effect modification Similar associations were observed for total apoC-III across all subgroups in stratified analyses (ESM Fig. 2a). Associations for HDL lacking apoC-III with incident diabetes tended to be more strongly inverse in lower risk subgroups (BMI $<25 \mathrm{~kg} / \mathrm{m}^{2}$, non-smokers, metabolically healthy individuals), although none of the subgroup differences were significant (ESM Fig. 2b). Similar to the overall association, associations for HDL containing apoC-III were null across all subgroups. No differences in associations were observed for either HDL subspecies by sex or across racial/ ethnic groups; however, numbers were limited for the evaluation of effect modification by race/ethnicity (with only 68 diabetes cases among Chinese-Americans, 174 cases among African-Americans and 158 cases among Hispanics).

\section{Discussion}

In a multi-ethnic US cohort of men and women, elevated levels of plasma apoC-III were associated with a substantially higher rate of diabetes, with a nearly twofold difference in incidence between extreme quintiles. ApoC-III also eliminated the protective association of HDL with incidence of diabetes; concentrations of HDL lacking apoC-III were associated with lower incidence, while HDL containing apoC-III was not associated. Supporting these findings, total apoC-III was positively associated with fasting plasma glucose and $\mathrm{HbA}_{1 \mathrm{c}}$ and inversely associated with insulin sensitivity, and only HDL lacking apoC-III was beneficially associated with these measures. In the subset of participants with plasma apoB measures, no association with incident diabetes was present for either LDL containing or lacking apoC-III, and while associations did not vary significantly between the two VLDL subspecies, VLDL exhibited a stronger (non-significant) positive association with incident diabetes in the absence of apoC-III. Associations for total apoC-III and apoC-III-defined lipoprotein subspecies were accounted for by plasma triacylglycerols, a hypothesised intermediate in the pathway between apoC-III levels and incident diabetes. 
Table 3 HRs and 95\% CIs for incidence of diabetes per SD of plasma LDL and VLDL measures in MESA

\begin{tabular}{|c|c|c|c|c|c|c|}
\hline & \multicolumn{3}{|l|}{ LDL $(n=1545)$} & \multicolumn{3}{|l|}{$\operatorname{VLDL}(n=1526)$} \\
\hline & Total LDL & LDL lacking apoC-III & $\begin{array}{l}\text { LDL containing } \\
\text { apoC-III }\end{array}$ & Total VLDL & $\begin{array}{l}\text { VLDL lacking } \\
\text { apoC-III }\end{array}$ & $\begin{array}{l}\text { VLDL containing } \\
\text { apoC-III }\end{array}$ \\
\hline $\mathrm{SD}(\mathrm{g} / \mathrm{l})$ & 0.18 & 0.17 & 0.01 & 0.02 & 0.01 & 0.005 \\
\hline Crude model & $1.06(0.95,1.17)$ & $1.05(0.94,1.18)$ & $1.00(0.88,1.14)$ & $1.23(1.11,1.36)$ & $1.20(1.02,1.41)$ & $1.03(0.88,1.21)$ \\
\hline Multivariable model & $1.01(0.91,1.12)$ & $1.00(0.89,1.12)$ & $1.03(0.90,1.17)$ & $1.10(0.97,1.24)$ & $1.15(0.97,1.37)$ & $1.01(0.85,1.19)$ \\
\hline
\end{tabular}

HRs obtained from weighted Cox proportional hazard regression models in the case-cohort subset with LDL and VLDL measures. LDL and VLDL exposures (assessed via concentrations of apoB) were log-transformed in analyses. The $p_{\text {trend }}$ uses the medians of (log-transformed) apolipoprotein variables

Crude models stratified by age and sex and adjusted for race. Multivariable models additionally adjusted for smoking, income, alcohol intake, BMI, systolic BP and anti-hypertensive medication use

$p_{\text {het }}$, LDL containing and lacking apoC-III: crude model, $p=0.36$; multivariable model, $p=0.99$

$p_{\text {het }}$, VLDL containing and lacking apoC-III: crude model, $p=0.82$; multivariable model, $p=0.66$

Our findings in the ethnically diverse MESA cohort demonstrate that the positive associations for total apoC-III [15, $16]$ with incident diabetes previously reported by our group [15] and others [16] in predominantly white cohorts extend to other racial/ethnic populations. We did not detect significant effect modification by race/ethnicity for any of our associations in MESA (all $p$-interaction $\geq 0.2$ ). We previously reported divergent associations for apoC-III-defined HDL subspecies in the Danish Diet, Cancer and Health study [15]; however, this was a secondary analysis in a case-cohort designed for the analysis of CHD, and biochemical measures of glucose metabolism were not available to investigate potential mechanistic links between apoC-III-defined HDL subspecies and diabetes. Furthermore, by expanding our investigation of apoC-III-defined lipoprotein subspecies to LDL and VLDL, these analyses provide novel insight into the diabetogenic potential of apoC-III on specific lipoprotein entities.

ApoC-III plays a key role in lipoprotein metabolism by increasing levels of circulating triacylglycerols, primarily through delaying the clearance of triacylglycerol-rich lipoproteins [30, 31], but potentially also by promoting production of these lipoproteins [30, 31] and impairing triacylglycerol lipolysis [6-9]. Genetic evidence suggests that these triacylglycerol regulatory actions, believed to be largely responsible for the involvement of apoC-III in CVD, may also account for the association between total apoC-III and diabetes. Overexpression of the Apoc3 gene in mice results in elevated circulating triacylglycerol levels and increased susceptibility to hepatic insulin resistance and fatty liver disease [10]. APOC3 genetic variants in humans have been linked to higher levels of both circulating apoC-III and triacylglycerols in a number of studies [32-36], some of which also found these variants to be associated with insulin resistance and non-alcoholic fatty liver disease [33, 34]. The complete attenuation of total apoC-III estimates with triacylglycerol adjustment in our analyses supports an influence of apoC-III on the risk of diabetes primarily through triacylglycerol-related mechanisms. The diminished associations for total apoC-III with adjustment for VLDL, the primary carrier of plasma triacylglycerols, provide further evidence for a triacylglycerolmediated role of apoC-III on the risk of diabetes.

The positive associations of total apoC-III with longitudinally assessed glucose and $\mathrm{HbA}_{1 \mathrm{c}}$ concentrations and inverse association with insulin sensitivity are also consistent with a direct role of apoC-III in diabetes pathophysiology through the regulation of glucose and insulin levels. ApoC-III potentially promotes hepatic insulin resistance by disrupting insulin signalling via the activation of protein kinase $\mathrm{C}-\varepsilon$ in endothelial cells [10] and stimulates the apoptosis of insulin-secreting pancreatic beta cells through $\mathrm{Ca}^{2+}$-dependent mechanisms $[12,37]$. In addition, apoC-III may contribute to the development of diabetes through inflammatory mechanisms, as apoCIII has been shown to induce monocyte adhesion to endothelial cells and enhance the production of inflammatory molecules $[13,14]$. However, as adjustment for inflammatory markers had minimal influence on our estimates, our findings do not clearly support an inflammatory role of apoC-III in diabetes pathogenesis.

The current results and our prior findings $[15,18,38]$ indicate that, beyond its inherent atherogenic and diabetogenic properties, apoC-III may be involved in cardiometabolic disease processes as a modulator of HDL function. We previously reported that only HDL lacking apoC-III was inversely associated with risk of coronary heart disease in four cohorts [38] and with cross-sectional measures of subclinical atherosclerosis in MESA [18], and thus HDL containing apoC-III may constitute a dysfunctional subspecies of HDL lacking cardioprotective potential. Little is currently known about the functional involvement of these apoC-III-defined HDL subspecies in the development of diabetes. However, the present findings suggest that apoC-III may disrupt the increasingly recognised anti-diabetogenic actions of HDL, which include potentially wide-ranging effects on glucose homoeostasis via 
the promotion of glucose uptake in skeletal muscle, the regulation of insulin secretion and the enhancement of insulin sensitivity via anti-inflammatory mechanisms [2]. Consistent with a protective role of HDL in diabetes pathophysiology and in agreement with prior observational studies [15, 39-43], total HDL was beneficially associated with both incident diabetes and measures of glucose metabolism in our analyses. However, our investigation of apoC-III-defined HDL subspecies revealed that these protective associations are no longer present when HDL contains apoC-III. Although further biological studies are needed to investigate the precise mechanisms through which apoC-III might modulate the relationship between HDL and diabetes, our associations with continuous glycaemic measures support the hypothesis that apoC-III acts to inhibit the glucoregulatory and anti-insulinogenic properties of HDL.

In addition to its inherent proinflammatory effects [13, 14], apoC-III may promote the development of diabetes by suppressing HDL-mediated anti-inflammatory effects. Supporting this hypothesis, only HDL lacking apoC-III has been shown to inhibit monocyte adhesion to endothelial cells in vitro [14]. Although, as for total apoC-III, associations for apoC-IIIdefined HDL subspecies were essentially unchanged with adjustment for CRP and IL-6, further investigation of these associations in cohorts with additional inflammatory biomarkers might provide additional insight into the potential immunomodulatory actions of apoC-III on HDL function.

In contrast to the anti-diabetogenic actions of HDL, VLDL is believed to be involved in diabetes pathophysiology through the promotion of hypertriacylglycerolaemia [44], and more limited evidence suggests that LDL may also enhance the risk of diabetes by inhibiting the secretion of insulin [20] and decreasing pancreatic beta cell proliferation [20] and survival [45]. Although we speculated that the presence of apoC-III on LDL and VLDL might strengthen their associations with incident diabetes and fasting glucose, we found associations for LDL to be similarly null irrespective of the presence of apoC-III, and VLDL exhibited stronger (non-significant) positive associations when it lacked apoC-III. While our results for apoC-III-defined VLDL subspecies are seemingly at odds with the hypothesised diabetogenic role of apoC-III, these findings are consistent with our previous report in the Cholesterol and Recurrent Events trial of an adverse association with recurrent CHD only for VLDL lacking apoC-III [46]. The attenuation of VLDL estimates in triacylglycerol-adjusted models corresponds with the established role of VLDL as a key transporter of plasma triacylglycerols [47] and is also in agreement with two prior small cross-sectional studies that found no differences in levels of VLDL containing apoC-III [48] or apoCIII in VLDL [49] by diabetes status among participants with similar triacylglycerol levels.

Strengths of our study include the relatively large number of cases and long, sustained follow-up. Our comprehensive exposure and outcome assessment provided the opportunity to conduct an in-depth analysis of the potential diabetogenic role of apoC-III on different lipoprotein classes. The inclusion of four racial/ethnic groups in MESA allowed us to generalise results beyond the predominantly white populations included in prior studies. However, our study has a number of notable limitations. The availability of apolipoprotein measures only at baseline precluded our ability to examine whether changes in apolipoprotein levels might be related to risk of diabetes. Although our use of a novel ELISA presumably enhanced the accuracy of our apolipoprotein measurements, it is possible that associations were underestimated due to measurement error. In addition, numbers were limited for the assessment of potential effect modification in subgroup analyses and for the evaluation of associations for LDL and VLDL subspecies in the case-cohort subset with available measurements.

In conclusion, our findings support an adverse association between apoC-III and risk of diabetes, potentially via the enhancement of circulating triacylglycerol levels and the disruption of glucose homoeostasis. The absence of an inverse association for HDL containing apoC-III also suggests that apoC-III may detrimentally impact on the protective functions of HDL. Continued research into apoC-III and apoC-IIIdefined lipoprotein subspecies will provide further insight into the biological underpinnings of these associations and may inform the development of novel diabetes treatment and prevention strategies targeting lipid-based pathways.

Acknowledgements The authors thank the MESA investigators, staff and participants for their valuable contributions. A full list of participating MESA investigators and institutions can be found at http://www.mesanhlbi.org.

Data availability The data, analytical methods and study materials will be made available to other researchers for the purposes of reproducing the results only with proper IRB approvals and strict adherence to cohortspecific regulations. Requests for data can be directed to RLM or MKJ.

Funding MESA is a National Heart, Lung, and Blood Institute (NHLBI)-funded study supported by grants R01 HL071739 and R21 HL091217 from the NHLBI, T32 DK 007703 from the National Institute of Diabetes and Digestive and Kidney Diseases, UL1-TR000040 and UL1-TR-001079 from the National Centre for Research Resources, and by contracts N01-HC-95159, N01-HC-95160, N01-HC95161, N01-HC-95162, N01-HC-95163, N01-HC-95164, N01-HC95165, N01-HC-95166, N01-HC-95167, N01-HC-95168 and N01-HC95169 from the NHLBI. The present study was supported by the ADA (grant no. 1-15-JF-30) and HDL apoC-III measurements were supported by an independent research grant from Roche Pharmaceuticals and by the William F. Milton Fund, Harvard Medical School.

Duality of interest Roche Pharmaceuticals provided unrestricted funding for the development of the novel apoA-I/apoC-III ELISA and had no role in the design of the study, data collection, analyses or report. Harvard University holds a patent for the measurement of HDL subspecies by apoC-III where MKJ, JDF and FMS are named co-inventors.

Contribution statement SAA drafted the manuscript, performed statistical analyses and interpreted the data with support from KJM and MKJ. 
JDF conducted the laboratory measurements, compiled the apolipoprotein dataset and drafted the ESM Methods. FMS and RLM provided valuable input on the study hypothesis and design, and FMS supervised laboratory measurements. MKJ conceived and designed the study and obtained funding for measures of HDL subspecies. MYT was involved in study design and data acquisition. All authors edited manuscript drafts, critically revised the manuscript for important intellectual content and gave final approval of the version to be published. MKJ is the guarantor of this work.

\section{References}

1. American Diabetes Association (2017) 2. Classification and diagnosis of diabetes. Diabetes Care 40(Supplement 1):S11-S24. https://doi.org/10.2337/dc17-S005

2. Drew BG, Rye KA, Duffy SJ, Barter P, Kingwell BA (2012) The emerging role of HDL in glucose metabolism. Nat Rev Endocrinol 8(4):237-245. https://doi.org/10.1038/nrendo.2011.235

3. Mooradian AD (2009) Dyslipidemia in type 2 diabetes mellitus. Nat Clin Pract Endocrinol Metab 5(3):150-159. https://doi.org/10. 1038/ncpendmet1066

4. Wyler von Ballmoos MC, Haring B, Sacks FM (2015) The risk of cardiovascular events with increased apolipoprotein CIII: a systematic review and meta-analysis. J Clin Lipidol 9(4):498-510. https:// doi.org/10.1016/j.jacl.2015.05.002

5. Zheng C, Khoo C, Furtado J, Sacks FM (2010) Apolipoprotein CIII and the metabolic basis for hypertriglyceridemia and the dense low-density lipoprotein phenotype. Circulation 121(15):17221734. https://doi.org/10.1161/CIRCULATIONAHA.109.875807

6. Sacks FM (2015) The crucial roles of apolipoproteins E and C-III in apoB lipoprotein metabolism in normolipidemia and hypertriglyceridemia. Curr Opin Lipidol 26(1):56-63. https://doi.org/10.1097/ MOL.0000000000000146

7. Brown WV, Baginsky ML (1972) Inhibition of lipoprotein lipase by an apoprotein of human very low density lipoprotein. Biochem Biophys Res Commun 46(2):375-382. https://doi.org/10.1016/ S0006-291X(72)80149-9

8. Liu H, Talmud PJ, Lins L et al (2000) Characterization of recombinant wild type and site-directed mutations of apolipoprotein C-III: lipid binding, displacement of ApoE, and inhibition of lipoprotein lipase. Biochemistry 39(31):9201-9212. https://doi.org/10.1021/ bi0009441

9. Wang CS, McConathy WJ, Kloer HU, Alaupovic P (1985) Modulation of lipoprotein lipase activity by apolipoproteins. Effect of apolipoprotein C-III. J Clin Invest 75(2):384-390. https://doi.org/10.1172/JCI111711

10. Lee HY, Birkenfeld AL, Jornayvaz FR et al (2011) Apolipoprotein $\mathrm{CIII}$ overexpressing mice are predisposed to diet-induced hepatic steatosis and hepatic insulin resistance. Hepatology 54(5):1650 1660. https://doi.org/10.1002/hep.24571

11. Avall K, Ali Y, Leibiger IB et al (2015) Apolipoprotein CIII links islet insulin resistance to $\beta$-cell failure in diabetes. Proc Natl Acad Sci U S A 112(20):E2611-E2619. https://doi.org/10.1073/pnas. 1423849112

12. Holmberg R, Refai E, Hoog A et al (2011) Lowering apolipoprotein CIII delays onset of type 1 diabetes. Proc Natl Acad Sci U S A 108(26):10685-10689. https://doi.org/10.1073/pnas.1019553108

13. Kawakami A, Aikawa M, Alcaide P, Luscinskas FW, Libby P, Sacks FM (2006) Apolipoprotein CIII induces expression of vascular cell adhesion molecule-1 in vascular endothelial cells and increases adhesion of monocytic cells. Circulation 114(7):681687. https://doi.org/10.1161/CIRCULATIONAHA.106.622514

14. Kawakami A, Aikawa M, Libby P, Alcaide P, Luscinskas FW, Sacks FM (2006) Apolipoprotein CIII in apolipoprotein B lipoproteins enhances the adhesion of human monocytic cells to endothelial cells. Circulation 113(5):691-700. https://doi.org/10. 1161/CIRCULATIONAHA.105.591743

15. Aroner SA, Yang M, Li J et al (2017) Apolipoprotein C-III and high-density lipoprotein subspecies defined by apolipoprotein CIII in relation to diabetes risk. Am J Epidemiol 186(6):736-744. https://doi.org/10.1093/aje/kwx143

16. Onat A, Hergenc G, Ayhan E et al (2009) Serum apolipoprotein CIII in high-density lipoprotein: a key diabetogenic risk factor in Turks. Diabet Med 26(10):981-988. https://doi.org/10.1111/j. 1464-5491.2009.02814.x

17. Jensen MK, Aroner SA, Mukamal KJ et al (2017) HDL subspecies defined by presence of apolipoprotein C-III and incident coronary heart disease in four cohorts. Circulation 137:1364-1373

18. Aroner SA, Koch M, Mukamal KJ et al (2018) High-density lipoprotein subspecies defined by apolipoprotein C-III and subclinical atherosclerosis measures: MESA (the Multi-Ethnic Study of Atherosclerosis). J Am Heart Assoc 7:e007824

19. Mendivil CO, Rimm EB, Furtado J, Chiuve SE, Sacks FM (2011) Low-density lipoproteins containing apolipoprotein C-III and the risk of coronary heart disease. Circulation 124(19):2065-2072. https://doi.org/10.1161/CIRCULATIONAHA.111.056986

20. Rutti S, Ehses JA, Sibler RA et al (2009) Low- and high-density lipoproteins modulate function, apoptosis, and proliferation of primary human and murine pancreatic $\beta$-cells. Endocrinology 150(10):4521-4530. https://doi.org/10.1210/en.2009-0252

21. Mora S, Otvos JD, Rosenson RS, Pradhan A, Buring JE, Ridker PM (2010) Lipoprotein particle size and concentration by nuclear magnetic resonance and incident type 2 diabetes in women. Diabetes 59(5):1153-1160. https://doi.org/10.2337/db09-1114

22. Mackey RH, Mora S, Bertoni AG et al (2015) Lipoprotein particles and incident type 2 diabetes in the Multi-Ethnic Study of Atherosclerosis. Diabetes Care 38(4):628-636. https://doi.org/10. 2337/dc14-0645

23. Bild DE, Bluemke DA, Burke GL et al (2002) Multi-Ethnic Study of Atherosclerosis: objectives and design. Am J Epidemiol 156(9): 871-881. https://doi.org/10.1093/aje/kwf113

24. McAuley KA, Williams SM, Mann JI et al (2001) Diagnosing insulin resistance in the general population. Diabetes Care 24(3): 460-464. https://doi.org/10.2337/diacare.24.3.460

25. Expert Committee on the Diagnosis and Classification of Diabetes Mellitus (2003) Report of the Expert Committee on the Diagnosis and Classification of Diabetes Mellitus. Diabetes Care 26(Suppl 1): S5-S20

26. Petersen L, Sorensen TI, Andersen PK (2003) Comparison of casecohort estimators based on data on premature death of adult adoptees. Stat Med 22(24):3795-3803. https://doi.org/10.1002/ sim. 1672

27. Kalbfleisch JD, Prentice RL (1980) The statistical analysis of failure time data. Wiley, Chichester

28. Kohan AB (2015) Apolipoprotein C-III: a potent modulator of hypertriglyceridemia and cardiovascular disease. Curr Opin Endocrinol Diabetes Obes 22(2):119-125. https://doi.org/10.1097/ MED.0000000000000136

29. Grundy SM, Brewer HB Jr, Cleeman JI et al (2004) Definition of metabolic syndrome: report of the National Heart, Lung, and Blood Institute/American Heart Association conference on scientific issues related to definition. Circulation 109(3):433-438. https://doi. org/10.1161/01.CIR.0000111245.75752.C6

30. Qin W, Sundaram M, Wang Y et al (2011) Missense mutation in APOC3 within the C-terminal lipid binding domain of human ApoC-III results in impaired assembly and secretion of triacylglycerol-rich very low density lipoproteins: evidence that ApoC-III plays a major role in the formation of lipid precursors within the microsomal lumen. J Biol Chem 286(31):2776927780. https://doi.org/10.1074/jbc.M110.203679 
31. Sundaram M, Zhong S, Bou Khalil M et al (2010) Expression of apolipoprotein C-III in McA-RH7777 cells enhances VLDL assembly and secretion under lipid-rich conditions. J Lipid Res 51(1): 150-161. https://doi.org/10.1194/jlr.M900346-JLR200

32. Jorgensen AB, Frikke-Schmidt R, Nordestgaard BG, TybjaergHansen A (2014) Loss-of-function mutations in APOC3 and risk of ischemic vascular disease. N Engl J Med 371(1):32-41. https:// doi.org/10.1056/NEJMoa1308027

33. Peter A, Kantartzis K, Machicao F et al (2012) Visceral obesity modulates the impact of apolipoprotein $\mathrm{C} 3$ gene variants on liver fat content. Int J Obes 36(6):774-782. https://doi.org/10.1038/ijo. 2011.154

34. Petersen KF, Dufour S, Hariri A et al (2010) Apolipoprotein C3 gene variants in nonalcoholic fatty liver disease. N Engl J Med 362(12):1082-1089. https://doi.org/10.1056/NEJMoa0907295

35. Pollin TI, Damcott CM, Shen H et al (2008) A null mutation in human APOC3 confers a favorable plasma lipid profile and apparent cardioprotection. Science 322(5908):1702-1705. https://doi. org/10.1126/science. 1161524

36. TG and HDL Working Group of the Exome Sequencing Project, National Heart, Lung, and Blood Institute, Crosby J, Peloso GM, Auer PL, Reiner AP (2014) Loss-of-function mutations in APOC3, triglycerides, and coronary disease. N Engl J Med 371:22-31

37. Juntti-Berggren L, Refai E, Appelskog I et al (2004) Apolipoprotein $\mathrm{CIII}$ promotes $\mathrm{Ca}^{2+}$-dependent $\beta$ cell death in type 1 diabetes. Proc Natl Acad Sci U S A 101(27):10090-10094. https://doi.org/10. 1073/pnas.0403551101

38. Jensen MK, Rimm EB, Furtado JD, Sacks FM (2012) Apolipoprotein C-III as a potential modulator of the association between HDLcholesterol and incident coronary heart disease. J Am Heart Assoc 1: $\mathrm{e} 000232$

39. Abbasi A, Corpeleijn E, Gansevoort RT et al (2013) Role of HDL cholesterol and estimates of HDL particle composition in future development of type 2 diabetes in the general population: the PREVEND study. J Clin Endocrinol Metab 98(8):E1352-E1359. https://doi.org/10.1210/jc.2013-1680

40. Haase CL, Tybjaerg-Hansen A, Nordestgaard BG, Frikke-Schmidt R (2015) HDL cholesterol and risk of type 2 diabetes: a Mendelian randomization study. Diabetes 64(9):3328-3333. https://doi.org/10. 2337/db14-1603
41. Njolstad I, Arnesen E, Lund-Larsen PG (1998) Sex differences in risk factors for clinical diabetes mellitus in a general population: a 12-year follow-up of the Finnmark study. Am J Epidemiol 147(1): 49-58. https://doi.org/10.1093/oxfordjournals.aje.a009366

42. von Eckardstein A, Schulte H, Assmann G (2000) Risk for diabetes mellitus in middle-aged Caucasian male participants of the PROCAM study: implications for the definition of impaired fasting glucose by the American Diabetes Association. J Clin Endocrinol Metab 85(9):3101-3108. https://doi.org/10.1210/jcem.85.9.6773

43. Wilson PW, Meigs JB, Sullivan L, Fox CS, Nathan DM, D'Agostino RB Sr (2007) Prediction of incident diabetes mellitus in middle-aged adults: the Framingham Offspring study. Arch Intern Med 167(10):1068-1074. https://doi.org/10.1001/archinte. 167.10.1068

44. Sparks JD, Sparks CE, Adeli K (2012) Selective hepatic insulin resistance, VLDL overproduction, and hypertriglyceridemia. Arterioscler Thromb Vasc Biol 32(9):2104-2112. https://doi.org/ 10.1161/ATVBAHA.111.241463

45. Roehrich ME, Mooser V, Lenain V et al (2003) Insulin-secreting $\beta$ cell dysfunction induced by human lipoproteins. J Biol Chem 278(20):18368-18375. https://doi.org/10.1074/jbc.M300102200

46. Lee SJ, Campos H, Moye LA, Sacks FM (2003) LDL containing apolipoprotein $\mathrm{CIII}$ is an independent risk factor for coronary events in diabetic patients. Arterioscler Thromb Vasc Biol 23(5):853-858. https://doi.org/10.1161/01.ATV.0000066131.01313.EB

47. Havel RJ (1989) Biology of cholesterol, lipoproteins and atherosclerosis. Clin Exp Hypertens A 11(5-6):887-900. https://doi.org/ 10.3109/10641968909035380

48. Lee SJ, Moye LA, Campos H, Williams GH, Sacks FM (2003) Hypertriglyceridemia but not diabetes status is associated with VLDL containing apolipoprotein CIII in patients with coronary heart disease. Atherosclerosis 167(2):293-302. https://doi.org/10. 1016/S0021-9150(03)00007-8

49. Ishibashi S, Yamada N, Shimano H, Takaku F, Akanuma Y, Murase $\mathrm{T}$ (1989) Composition of very-low-density lipoproteins in noninsulin-dependent diabetes mellitus. Clin Chem 35(5):808-812

Publisher's note Springer Nature remains neutral with regard to jurisdictional claims in published maps and institutional affiliations. 